\title{
ESDA2010-व०स००
}

\section{ENTROPY BASED CLASSIFICATION OF ROAD-PROFILES}

\author{
S. Çağlar Başlamışı \\ Department of Mechanical Engineering \\ Hacettepe University \\ Beytepe, Ankara 06800 \\ Turkey \\ Email: scaglarb@hacettepe.edu.tr
}

\author{
Selis Önel \\ Department of Chemical Engineering \\ Hacettepe University \\ Beytepe, Ankara 06800 \\ Turkey \\ Email: selis@hacettepe.edu.tr
}

\begin{abstract}
In this study, the Shannon entropies of six different roadprofiles ranging from "very good asphalt road" to "dirt road (terrain)" were calculated. Results indicate that each type of road has a well defined entropy value and that the entropies of roads ranging from "very good asphalt" to "dirt road (terrain)" lie on a nearly linear locus. A second approach presented in this paper consists in measuring the sprung mass vertical acceleration of a vehicle running over segments of roads of different qualities and calculating the entropies of the acceleration signals. This procedure has been applied to assess the influence of nonlinear damping and vehicle speed. It has been seen that it is possible to identify the type of the road surface through the calculation of entropy if the vehicle operating parameters are known. Finally, it is observed that the locus of entropy values is concave on the plot of acceleration entropy versus root mean square (RMS) acceleration.
\end{abstract}

\section{KEYWORDS}

Shannon entropy, road roughness, road surface modeling

\section{NOMENCLATURE}

$\omega \quad$ Angular frequency $[\mathrm{rad} / \mathrm{s}]$

$V \quad$ Longitudinal vehicle velocity $[\mathrm{m} / \mathrm{s}]$

$\sigma^{2} \quad$ Variance of the road irregularities $\left[\mathrm{m}^{2}\right]$

$\alpha \quad$ Spatial cut-off frequency $\left[\mathrm{m}^{-1}\right]$

(A coefficient depending on the type of road surfaces)

$x_{u} \quad$ Unsprung mass vertical displacement [m]

$x_{s} \quad$ Unsprung mass vertical displacement [m]

$m_{s} \quad$ Sprung mass $[\mathrm{kg}]$

$m_{u} \quad$ Unsprung mass $[\mathrm{kg}]$ $k_{s} \quad$ Suspension stiffness [N/m]

$k_{u} \quad$ Tire stiffness $[\mathrm{N} / \mathrm{m}]$

$\mathrm{c}_{\mathrm{s}} \quad$ Suspension damping $[\mathrm{Ns} / \mathrm{m}]$

$\kappa \quad$ Constant factor determining the damping coefficient in bounce and rebound modes

$\omega_{s} \quad$ Sprung mass natural frequency, $\omega_{g}=\left(k_{g} / m_{g}\right)^{1 / 2}$

$\omega_{u} \quad$ Unsprung mass natural frequency,

$$
\omega_{u}=\left[\left(k_{s}+k_{u}\right) / m_{u}\right]^{1 / 2}
$$

$\xi_{g} \quad$ Sprung mass damping ratio, $\xi_{g}=c_{g} / 2\left(m_{g} k_{g}\right)^{1 / 2}$

$\rho_{s} \quad$ Sprung/unsprung mass ratio, $\rho_{s}=m_{g} / m_{u}$

$H(X) \quad$ Shannon entropy of random variable $X$

\section{INTRODUCTION}

Road roughness has been extensively studied in the literature for many reasons. First of all, road roughness directly affects vehicle ride comfort. Moreover, dynamic wheel loads that are generated by the roughness of the road deteriorate road surface quality and affect road maintenance costs. As far as bridge crossing is concerned, these loads must be taken into account at the design stage of the bridge as the deterioration of road surface quality -and resulting increase in dynamics wheel loads- are likely to endanger the stability of the structure. A typical road-profile is characterized by the existence of large isolated irregularities, such as potholes or bumps, which are superposed to smaller but continuously distributed profile irregularities. For the purposes of the present study, only the latter type of road irregularities is considered.

A substantial number of indices have been proposed so far for the measurement of road roughness. Response-type road roughness measuring (RTRRM) systems measure the 
cumulative relative displacement between the axle and the vehicle body and average those displacements over some distance of the roadway $(\mathrm{mm} / \mathrm{km})$. RTRRMs are reported to not correlate well with user response to roadway roughness. Profile-based indices are obtained by either simulating the response of an RTRRM system as it traverses the profile or by filtering and weighting the spectra of wavebands that make up the road surface profile. Profile based indices, such as the International Roughness Index (IRI), the Ride Number $\left(\mathrm{RN}_{\text {Sayers }}\right.$ and $\mathrm{RN}_{\text {Janoff }}$ ), and the Ride Quality Index (RQI), were reported to correlate well with drivers' perception of ride comfort [1].

In the literature, random road-profiles have often been considered as stochastic processes [2]. More precisely, extensive research studies have been dedicated for the determination of accurate power spectral density (PSD) representations of road-profiles resulting in the formulation of international standards. An example of such a standard is the ISO 8608 [3], which classifies road-profiles from "very good" to "very poor" according to their power spectral density. Meanwhile in the literature, there has been only one attempt concerning the analysis of road roughness using the entropy concept [4], which is an analysis tool originating from information theory that has found important applications in stochastic analysis and signal processing.

In this study, the entropies of six different road-profiles ranging from "very good asphalt road" to "dirt road (terrain)" have been calculated. The procedure for the calculation of the entropies encompasses four steps that can be listed as:

1) Constructing a road-profile satisfying the power spectral density relation by adequately filtering a white noise input,

2) Obtaining the histogram of the road-profile to assess the distribution of road elevations along the road segment,

3) Obtaining the probability mass function of the former distribution of road elevations, and finally,

4) Calculating the Shannon entropy of the road-profile.

Another analysis approach employed in this study consists in measuring the sprung mass vertical acceleration of the vehicle running over segments of roads of different qualities and applying the four-step procedure for calculating the entropies of the acceleration signals. The procedure has been applied many times to assess the influence of changing vehicle operating parameters. It has been seen that it is possible to identify the type of the road surface through the calculation of the entropy values for a vehicle, whose operating parameters are known.

\section{ENTROPY AND SHANNON ENTROPY CONCEPTS}

The concept of entropy has applications starting from classical thermodynamics in physics and chemistry and extending into probability theory in statistics and mathematics, and not being limited to the information theory including the communication theory in electrical engineering, Kolmogorov complexity in computer science, investment theory in economics, and Occam's razor principle in philosophy of science [5]. Concept of entropy appeared with the second law of thermodynamics, formed the basis of statistical mechanics and expanded by the formation of information theory. It is difficult to assign a physical significance or physical quality to the concept of entropy from both the second law of thermodynamics developed using classical thermodynamics arguments or the information theory developed using statistical arguments [6]. In fact, entropy needs no interpretation as it is being used by the different disciplines of the scientific community as a tool for consistent reasoning of difficult and complex problems providing simple answers.

The concept of entropy was first introduced by R. Clausius $[7,8]$ in terms of heat to identify an additional thermodynamic state variable to quantify the qualitative experimental observation that all spontaneous processes occurring in an isolated constant-volume system result in the evolution of the system to a state of equilibrium [9]. This phenomenological concept of entropy suggests that the entropy change of an isolated system cannot be negative for any process and can at least be zero for reversible processes [10].

The atomistic or statistical concept of entropy first developed by L. Boltzmann and J. W. Gibbs consists in modeling of the energetic function entropy using the probability theory to express disorder in a quantitative way through a quantity $\Omega$ as:

$$
S=k \ln \Omega
$$

where $k$ is the Boltzmann constant and $\Omega$ represents the number of microstates corresponding to a given macrostate [11]. Boltzmann entropy provides the correlation between the concepts of disorder and entropy and is generalized by Gibbs, who defined entropy over a statistical ensemble of $N_{0}$ particles, i.e. over the probability distribution of various macrostates of a system [12] given by:

$$
S=-k N_{0} \sum_{i=1}^{r} P_{i} \ln P_{i}
$$

where $P_{i}$ is the probability that a particle lies in the $i^{\text {th }}$ macrostate. Both Boltzmann and Gibbs entropies form the foundation of statistical mechanics and are the basis of all the entropy concepts in modern physics. The entropy concept as a measure of information developed by C. E. Shannon [13] using statistical arguments also leads to the Boltzmann and Gibbs entropies.

In information theory, the concept of entropy can be interpreted through the Shannon axioms as a measure of the uncertainty in a probability distribution. Shannon axioms are defined for discrete probability distributions rather than continuous ones, but they can also be applied to continuous distributions [14]. Probability $P(A)$ of an event $A$ can be interpreted as a measure of uncertainty about the occurrence or nonoccurrence of $A$ in a single application of an experiment. The cases when $P(A)$ of an event is equal to 0.9 or 0.1 indicate almost certainty about the occurrence or nonoccurrence of that event, respectively. The uncertainty about event $A$ would be 
maximum if $P(A)$ is equal to 0.5 . Thus, this heuristic entropy could be interpreted as the measure of uncertainty of the occurrence or nonoccurrence of any event in a system prior to the execution of the underlying experiment [15]. The entropy of a discrete random variable $X$ with a probability mass function $p(x)=\operatorname{Pr}\{X=x\}, x \in X_{x}$ is defined as:

$$
H(X)=-\sum_{x} p(x) \log _{2} p(x)
$$

where $x$ defines the state in which $X$ resides, i.e. $p(x)$ is the probability that $X$ is in the state of $x$. The entropy is defined as zero when this probability is equal to zero. Thus, entropy does not depend on the actual values taken by the random variable $X$, but only on its probabilities. Logarithms to base 2 are used to measure the average uncertainty in the random variable in units of bits. Therefore, entropy can be interpreted in terms of information as the number of bits on average required to describe the random variable [5].

If $X$ is a continuous random variable, then the differential Shannon entropy is applied to continuous probability distributions of the random variable $X$, and is defined as:

$$
H(X)=-\int f(x) \log _{2} f(x) d x
$$

where $f(x)$ is the probability density function. Differential form of the Shannon entropy may be interpreted as a measure of the extent to which the distribution of the continuous random variable $X$ is concentrated over a small range of values or dispersed over a wide range of values, or, in other words, as a measure of the degree of indeterminacy of $X$ represented by its distribution [16].

The concept of entropy in information theory is related to the concept of entropy in statistical mechanics through the asymptotic equipartition property, which shows that the probability of a typical sequence is about $2^{-n H(X)}$ and there are about $2^{n H(X)}$ such sequences. In fact, using the probability distributions, it can be deduced from Eqn. (2) and Eqn. (3) that, as the same mathematical expression $-\Sigma p_{i} \log p_{i}$ appears in both equations, the thermodynamic entropy is identical to the information-theory entropy except for the presence of the Boltzmann constant [17]. The presence of such a relation is necessary, but not sufficient in itself to form a connection between these two fields, so that information theory can be applied to justify problems involving discrete random data, which cannot be handled by a statistical mechanics or a phenomenological thermodynamics approach due to the type of data. Studies [14, 17, 18] using the additive property and the concavity of the Shannon entropy have successfully showed that this concept can be used to relate some random data from a system to more important physical properties or information about a system.
Table 1. ROAD SURFACE TYPE PARAMETERS [20]

\begin{tabular}{lcc}
\hline \multicolumn{1}{c}{ Type of Road } & $\boldsymbol{\alpha \cdot \mathbf { 1 0 } ^ { \mathbf { 3 } } [ \mathrm { m } ^ { - 1 } ]}$ & ${\boldsymbol{\boldsymbol { \sigma } ^ { 2 }}}^{\mathbf{1} \mathbf{1 0}^{\mathbf{6}}\left[\mathrm{m}^{2}\right]}$ \\
\hline Road 1: Very Good Asphalt & 150 & 9 \\
Road 2: Good Asphalt & 225 & 44 \\
Road 3: Average Asphalt & 300 & 105 \\
Road 4: Poor Asphalt & 375 & 190 \\
Road 5: Paved Road & 450 & 300 \\
Road 6: Dirt Road (Terrain) & 750 & 750 \\
\hline
\end{tabular}

\section{ROAD SURFACE MODELING}

According to previous investigations on the subject [2], the displacement power spectral density of typical roads fits sufficiently accurately a piecewise linear curve in the logarithmic scale. This is also known as the split power approximation. The problem with the split power approximation is that it cannot be generated by linear shape filters [19]. Hence, it is not suitable for simulating the response of the vehicle. For a variety of road and terrain inputs, a good approximation of power spectral density given as a rational function of reasonably low order [20] is shown as:

$$
S(\omega)=\frac{\sigma^{2}}{\pi} \frac{\alpha V}{\omega^{2}+(\alpha V)^{2}}
$$

In this study, six different road-profiles, whose power spectral density functions are in the form of Eqn. (5), are considered. Angular frequency and variance data [20] for these profiles are given in Table 1.

It is often desired to analyze a system with a white noise input rather than one subjected to a fully shaped spectral density function. In such a case, a shaping filter is used to obtain the power spectral density function of the actual input from white noise. Then, by combining the filter equations with the equations of motion of the vehicle, a new system with white noise input can be obtained and analyzed. This procedure is applied in what follows.

Hac [20] gives the filter equation corresponding to the previously mentioned road surface model as:

$$
\frac{d}{d t} w(t)=-\alpha V w(t)+\eta(t)
$$

where $w(t)$ is the road surface displacement in meters to be solved, $V$ the forward vehicle velocity in $\mathrm{m} / \mathrm{s}$, and $\eta(t)$ the zero mean white noise in meters. In other words, $\eta(t)$ is the white noise input to the filter and $w(t)$ is the output of the filter. The 
relation between the power spectral density functions of the input and output of a system is given by:

$$
S_{\text {output }}(\omega)=(|H(\omega)|)^{2} S_{\text {input }}(\omega)
$$

where $H(\omega)$ is the complex frequency response function for the filter, which is derived in what follows.

Given the first order linear filter Eqn. (6) and letting $\xi(t)=e^{i \omega t}$, where $\omega$ is the frequency in $\mathrm{rad} / \mathrm{s}$, one can write $w(t)=H(\omega) e^{i \omega t}$. Differentiating both sides of the equation gives:

$$
\frac{d}{d t} w(t)=i \omega H(\omega) e^{i \omega t}
$$

Substituting Eqn. (8) in the filter equation gives:

$$
i \omega H(\omega) e^{i \omega t}=-\alpha V H(\omega) e^{i \omega t}+e^{i \omega t}
$$

from where $H(\omega)$ and its square are obtained as:

$$
H(\omega)=\frac{1}{i \omega+\alpha V}
$$

and

$$
(|H(\omega)|)^{2}=\frac{1}{\omega^{2}+(\alpha V)^{2}}
$$

Letting $S_{\text {input }}(\omega)=S_{0}$, where $S_{0}$ is the constant power spectral density function of the white noise process, and combining equations Eqn. (5) and Eqn. (11) gives:

$$
\frac{\sigma^{2}}{\pi} \frac{\alpha V}{\omega^{2}+(\alpha V)^{2}}=\frac{S_{0}}{\omega^{2}+(\alpha V)^{2}}
$$

Using Eqn. (12), $S_{0}$ is obtained as:

$$
S_{0}=\frac{\sigma^{2} \alpha V}{\pi}
$$

Finally, it is necessary to obtain a white noise with constant power spectral density $S_{0}$. A zero mean random data $z(k)$ with a variance $\sigma^{2}$ can be obtained from another zero mean random data $x(k)$ with unity variance if random data $x(k)$ is multiplied by $\sigma$. Thus, $z(k)=\sigma x(k)$ has variance $\sigma^{2}$ [21]. However, it is desired to obtain a white noise with a PSD level given by Eqn. (13). Accordingly, the white noise produced by a random number generator should be multiplied by the square root of
Eqn. (13) so as to obtain white noise with a PSD level of $S_{0}$. As a consequence, the desired white noise is obtained as:

$$
\eta(t)=\sqrt{\frac{\sigma^{2} \alpha V}{\pi}} \mathscr{N}(0,1)
$$

where $\mathcal{N}(0,1)$ gives $N$ data points with zero mean and unity variance $\sigma^{2}$.

\section{ROAD SURFACE CLASSIFICATION BASED ON DIRECT ROAD ELEVATION MEASUREMENTS}

The following procedure is implemented to compute the Shannon entropy of a given road surface. We assume the availability of an on-board measurement system collecting data at ordinary traffic speeds.

\section{Construction of road data}

In the literature, the profile of a given road type is often considered as ergodic [2]. Hence, we assume that a measurement system collecting data through different parallel paths along the road would return similar statistical properties for each path. Thus, it is sufficient to construct a single profile of road elevations according to Eqn. (6) for each road type. Determining the size of the data to be collected depends on various restrictions. Simple calculations based on the selection of an effective bandwidth according to the spatial cut-off frequency and a sampling frequency $\left(f_{s}=100 \mathrm{~Hz}\right)$, based on the fact that in such studies the largest frequency of interest is in the order of $50 \mathrm{~Hz}$, formed the grounds of the decision to choose a measurement length of 10 seconds and a data size of $N=1,000$. To be on the conservative side, a second set of calculations was also performed for $N=10,000$ for all types of roads to investigate the effect of data size on the computed value of entropy.

\section{Construction of the histogram of the road data}

A histogram of the data needs to be produced before obtaining the probability mass function of this data. Basically, this serves to separate data into adjacent bins each representing a range of road elevations and to compute the frequency distribution of data in each bin. The range of data to be considered for each bin of the histogram depends on the accuracy of the measurement system. In this study, we have assumed that this accuracy is in the range of $0.1 \mathrm{~mm}$.

\section{Calculation of the probability mass function}

Once the histogram is obtained, the number of data in each bin is divided by the size of data in order to assign a probability value to each bin. 


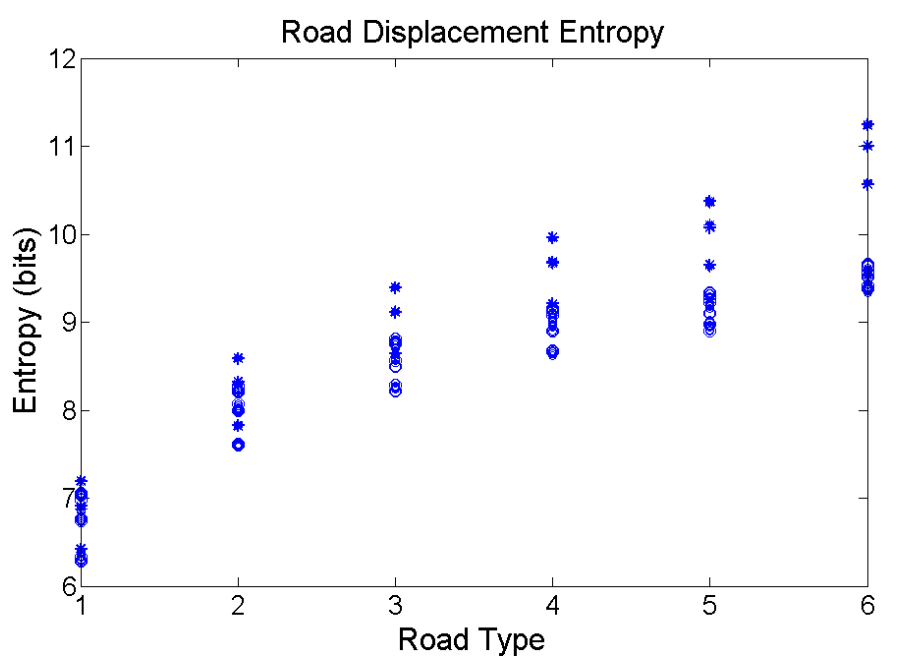

Figure 1. ENTROPY VALUES CALCULATED ON SIX DIFFERENT ROAD-PROFILES AT THREE DIFFERENT SPEEDS. "o": $N=1,000$; "*”: $N=10,000$.

\section{Calculation of Shannon's entropy}

Equation (3) is applied to compute the entropy of each road surface type. In this paper, a random number generator is employed in generating a normal distribution of data, which is subsequently processed through Eqn. (6) to Eqn. (14). To cope with the inherent inefficiency of this generator in yielding data with the exact desired frequency distribution and given that, for practical purposes, we can only work with data of finite size, the entropy calculation procedure has to be repeated many times, each time with a new data set for each road surface type, hence each time yielding a different value of entropy for each road surface type. In this study, we show, through simulations, that each road surface type is represented by a characteristic value of Shannon entropy.

\section{Simulation Results}

We calculated the Shannon entropy of each road surface type using this procedure assuming that the instrumentation system travels consecutively at speeds of 10,20 and $30 \mathrm{~m} / \mathrm{s}$. Application of the entropy calculation procedure ten times for each tour of the vehicle gives the results presented in Figure 1, where sample sizes of $N=1,000$ and $N=10,000$ are both employed. It is observed that each road surface type is characterized by a specific value of entropy at a given speed. Moreover, as the quality of a road surface deteriorates, its entropy increases.

Using larger sizes of data resulted in more satisfactory values of entropy as it was expected. However, it was noted for the case where the vehicle travels over different types of surfaces that the continuous gathering of data from the beginning of the test may lead to erroneous conclusions on the
Table 2. ENTROPY VALUES CALCULATED ON SIX DIFFERENT ROAD-PROFILES AT THREE DIFFERENT SPEEDS FOR $N=1,000$

\begin{tabular}{ccccccc}
\hline $\begin{array}{c}\text { Speed } \\
(\mathbf{m} / \mathbf{s})\end{array}$ & Road 1 & Road 2 & Road 3 & Road 4 & Road 5 & Road 6 \\
\hline 10 & 6.4 & 7.6 & 8.3 & 8.7 & 9.0 & 9.4 \\
20 & 6.7 & 8.0 & 8.6 & 9.0 & 9.2 & 9.5 \\
30 & 7.0 & 8.2 & 8.7 & 9.1 & 9.4 & 9.6 \\
\hline
\end{tabular}

Table 3. ENTROPY VALUES CALCULATED ON SIX DIFFERENT ROAD-PROFILES AT THREE DIFFERENT SPEEDS FOR $N=10,000$

\begin{tabular}{ccccccc}
\hline $\begin{array}{c}\text { Speed } \\
(\mathbf{m} / \mathbf{s})\end{array}$ & Road 1 & Road 2 & Road 3 & Road 4 & Road 5 & Road 6 \\
\hline 10 & 6.4 & 7.7 & 8.6 & 9.2 & 9.6 & 10.6 \\
20 & 6.9 & 8.3 & 9.1 & 9.7 & 10.1 & 11.0 \\
30 & 7.3 & 8.6 & 9.4 & 10.0 & 10.4 & 11.3 \\
\hline
\end{tabular}

type of road surface. Hence, a large data size may be impractical if the vehicle is traveling over different road surfaces during data collection. This observation led us to investigate the reliability of conclusions that can be reached with a data of smaller size. Figure 1 indicates that the entropy estimates for $N=1,000$ for different road surface types are different from those obtained using $N=10,000$. Despite the smaller data size, it is still possible to distinguish between different road surface types. However, a larger data size allows for better distinguishing between traveling speeds. Results of Fig. 1 are gathered in Tables 2 and 3, which may be used to interpolate the calculated values of entropy to characterize road surfaces when the travelling speeds are available.

\section{ROAD SURFACE CLASSIFICATION BASED ON ACCELEROMETER MEASUREMENTS}

Another approach for classifying road surface types consists in measuring the sprung mass vertical acceleration of the vehicle running over segments of roads of different qualities and applying the procedure for calculating the entropies of the acceleration signals. For this purpose, we need to introduce a vehicle model and then proceed to the calculation of entropies for different operating conditions of the vehicle, namely vehicle operating mass, damping coefficient and speed. 


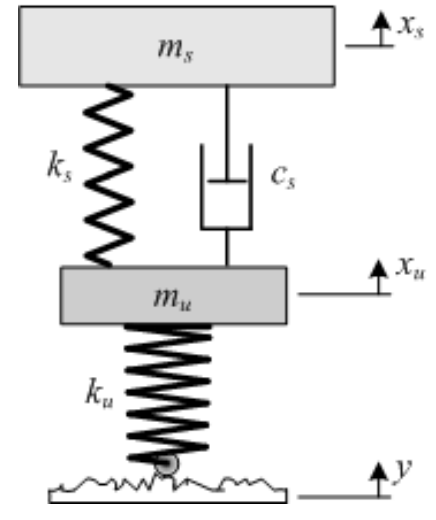

Figure 3. QUARTER CAR VEHICLE MODEL.

\section{Equations of Motion for the Vehicle Model}

Various vehicle models, such as the Quarter Car Vehicle Model, the Half Car Vehicle Model, and the Seven DOF Vehicle Model, are used by the vehicle dynamics community for the assessment of ride comfort. For passenger vehicles, the Quarter Car Vehicle Model shown in Figure 3 has been proven to model adequately the vehicle center of gravity (CG) bounce motion as long as road disturbance is small enough to assume linear suspension spring stiffness and therefore is used in this study for simplicity. by:

The equations of motion of the quarter car model are given

$$
\begin{aligned}
& \dot{x}_{1}=x_{2}-y \\
& \dot{x}_{2}=-\omega_{\mathrm{K}}^{2} x_{1}-2 \xi_{s}\left(1+\kappa \operatorname{sgn}\left(x_{\mathrm{a}}\right)\right) \omega_{\mathrm{s}} \rho_{\mathrm{g}} x_{2} \\
& +\omega_{s}^{2} \rho_{s} x_{2}+2 \xi_{s}\left(1+\kappa \operatorname{sgn}\left(x_{a}\right)\right) \omega_{a} \rho_{s} x_{4} \\
& \dot{x}_{2}=-x_{2}+x_{4} \\
& \dot{x}_{4}=2 \xi_{s}\left(1+\kappa \operatorname{sgn}\left(x_{a}\right)\right) \omega_{g} x_{2}-\omega_{g}^{2} x_{a} \\
& -2 \xi_{s}\left(1+\kappa \operatorname{sgn}\left(x_{a}\right)\right) \omega_{g} x_{4}
\end{aligned}
$$

where states are selected as $x_{1}=x_{u}-y, \quad x_{2}=\dot{x}_{u}$, $x_{\mathrm{a}}=x_{g}-x_{\mathrm{u}}$, and $x_{4}=x_{g}$.

The signals that can be processed for the classification of road surface entropy can be listed as:

- Sprung mass CG acceleration,

- Suspension stroke,

- Unsprung mass acceleration.

Given that real time integration is problematic due to inherent noise in the measurement system, it is generally preferred to work with the acceleration signal rather than the
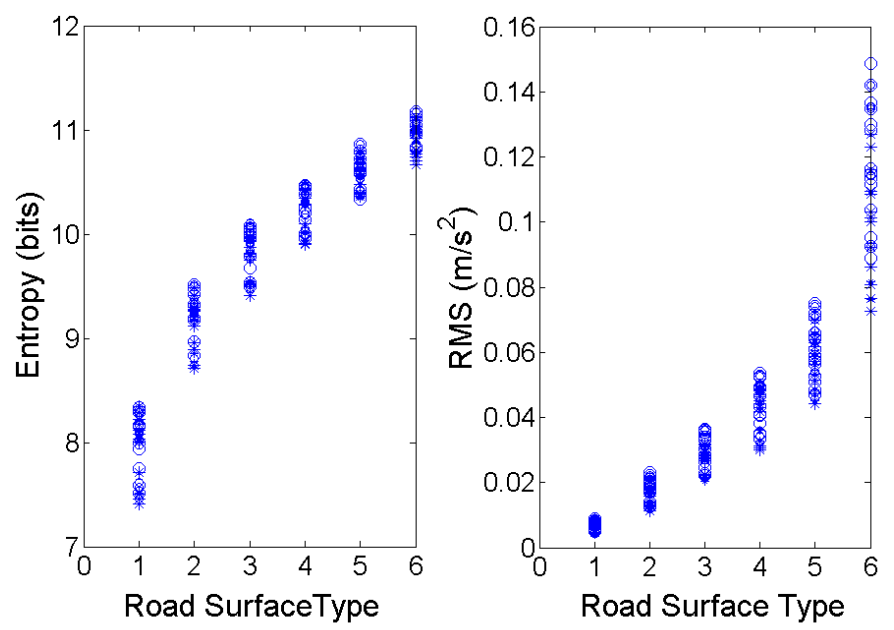

Figure 2. SPRUNG MASS ACCELERATION ENTROPY AND RMS VALUES CALCULATED ON SIX DIFFERENT ROADPROFILES AT THREE DIFFERENT SPEEDS AND FOR LINEAR "o": $\mathrm{k}=0.0$; AND NONLINEAR DAMPING “*”: $\mathrm{k}=0.5$. $(N=1,000)$.

velocity or the displacement. This precludes using suspension stroke for the calculation of entropy. Since modern vehicles are equipped with accelerometers mounted on their sprung mass, for practical purposes we use the sprung mass CG acceleration signal for the classification of road surfaces.

\section{Simulation Results}

Figure 3 shows the entropy and root mean square (RMS) values of sprung mass acceleration values calculated for linear and nonlinear vehicle models traveling over the six different road surface types at speeds of 10,20 and $30 \mathrm{~m} / \mathrm{s}$. The RMS values calculated for poor quality roads are scattered over a wide range as also suggested by the high entropy values calculated for the same road types. On the contrary, the RMS values for the good quality roads are densely collected around smaller values.

Tables 2 and 3 may be used to interpolate calculated entropy values to characterize road surfaces when the travelling speed is available. Results of Fig. 3 are combined in Fig. 4 where the independent variable -sprung mass acceleration RMS- can be considered as an indicator of the road surface quality. It is observed that as the quality of the road deteriorates, the expected increase of entropy follows a power law relationship of the form

$$
H\left(a_{\text {RMS }}\right)=-1.27 a_{\text {RMS }}-0.2954+13.5
$$

This relationship is valid for both the linear and nonlinear vehicle models running at different speeds on all six types of 


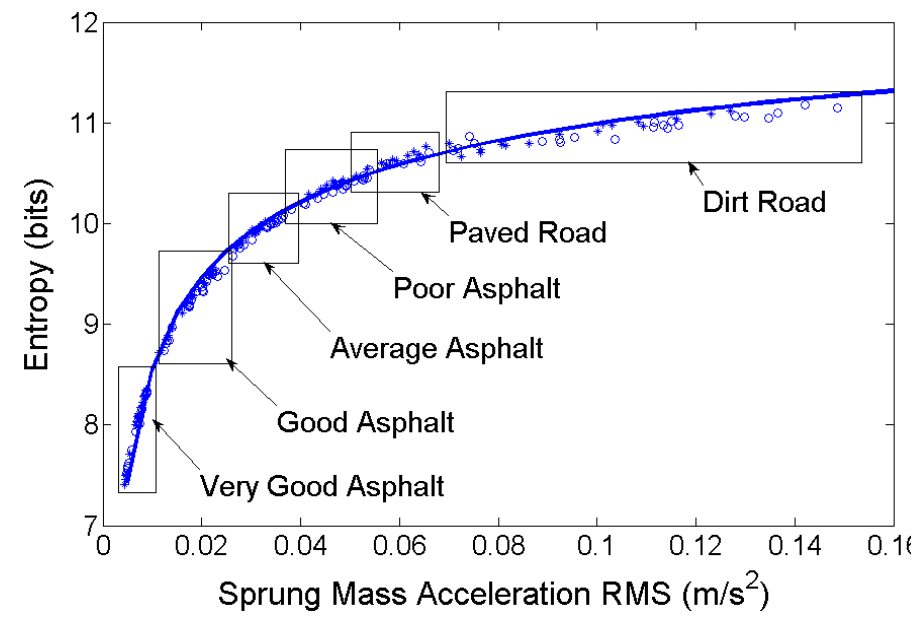

Figure 4. SPRUNG MASS ACCELERATION ENTROPY VS RMS CALCULATED ON SIX DIFFERENT ROAD-PROFILES AT THREE DIFFERENT SPEEDS FOR LINEAR "o": $\mathrm{k}=0.0$; AND NONLINEAR DAMPING “*”: $k=0.5(N=1,000)$.

road surfaces. Changing the sprung mass (loaded vehicle) also resulted in data falling on the same curve. The concave nature of the curve is typical of the general understanding of entropy. The following observations prevail:

1. Figure 4 serves the purpose of rating the quality of a roadprofile according to vehicle operating conditions (mass, speed, suspension characteristics) and measured vertical acceleration RMS. It is a clear indication of the intuitively supported fact that analyses based on the sole knowledge of road vertical displacement data (as suggested by many of the RTRRM indices) and without consideration of vehicle operating parameters can only yield a rough estimation of ride comfort. We have thus far observed that the increase of sprung mass, the decrease of vehicle speed, the decrease of suspension damping in compression mode all contribute to moving down the above mentioned curve. The analysis may be extended to include unsprung mass and tire stiffness. Furthermore, if similar curves are obtained for suspension stroke and tire deflection, a novel tool for passive suspension optimization may be obtained.

2. Entropy is a measure of uncertainty: If vehicle body bounce is perceived as a random event occurring as a result of some random input road signal, the increase in entropy should be understood as an increase in the chance of occurrence of a critical sequence of road displacement signals reducing ride comfort (corresponding to the human tolerance limits to vertical vibrations). In this respect, the rapid increase in entropy observed for a vehicle travelling on roads with good surface quality at increasing traveling speeds complies with an increase in the chance of occurrence of such disturbing signals.
3. There exist a one-to-one relationship between acceleration RMS and entropy. In this respect, the knowledge of any two of the following three parameters sets, i.e. acceleration RMS, operation condition, and entropy, fixes the other. For example, knowing the entropy and the RMS acceleration allows estimating the operating conditions. In general, suspension properties of the vehicle are known and the speed can be measured online. Thus, the mass of the sprung mass can be estimated.

\section{DISCUSSION OF RESULTS, CONCLUSIONS AND RECOMMENDATIONS FOR FUTURE WORK}

In the first part of this study, the Shannon entropies of six different road-profiles ranging from "very good asphalt road" to "dirt road (terrain)" were calculated. Results indicate that each type of road has a well defined entropy value at a given vehicle speed and that the entropies of roads ranging from "very good asphalt" to "dirt road (terrain)" lie on a nearly linear locus.

A second approach presented in this paper consists in measuring the sprung mass vertical acceleration of a vehicle running over segments of roads of different qualities and calculating the entropies of the acceleration signals. This procedure has been applied many times to assess the influence of vehicle operating parameters. It has been seen that it is possible to identify the type of the road surface and, more importantly, the decrease in ride comfort through the calculation of entropy for a vehicle with known operating parameters. An important observation is that the loci of acceleration entropy values versus acceleration RMS lie on a single concave curve, which contains all the required information related to the ride comfort of the vehicle interacting with different type of road surfaces.

Studies are currently under way to determine the shape of the curve shown in Fig. 4 for vehicles of different classes and undergoing non-stationary road events such as bump or pothole crossing.

\section{REFERENCES}

1. Heidenreich, W. and L. Hunt, Pavement Smoothness Indices Research Brief. 1998, Oregon Department of Transportation Technology Transfer Center: Oregon.

2. Dodds, C.J. and J.D. Robson, Description of Road SurfaceRoughness. Journal of Sound and Vibration, 1973. 31(2): p. 175-183.

3. Standardization, I.O.f., Mechanical Vibration - Road Surface Profiles - Reporting of Measured Data. 1995.

4. Andrei, R., The Use of Entropy Concept in Ranking and Correlating Roughness Measurements Obtained by Different Equipment in 2nd International Workshop on Artificial Intelligence and Mathematical Techniques in Pavement and Geomechanical Systems. 2000: Newark, DE. p. 153-164. 
5. Cover, T.M. and A.T. Joy, Elements of Information Theory. 2nd ed. 2006, Hoboken, New Jersey: John Wiley \& Sons, Inc.

6. Gaskell, D.R., Introduction to the Thermodynamics of Materials. 4th ed. 2003, New York: Taylor \& Francis Books, Inc.

7. Clausius, R., On the Moving Force of Heat, and the Laws Regarding the Nature of Heat Itself which are Deducible Therefrom. London, Edinburgh and Dublin Philosophical Magazine and Journal of Science, 1851. 2: p. 1-21, 102119.

8. Clausius, R., Über die bewegende Kraft der Wärme. Annalen der Physik, 1850. 79: p. 368-397, 500-524.

9. Sandler, S.I., Chemical and Engineering Thermodynamics. 2 ed. 1989, Toronto, Canada: John Wiley \& Sons, Inc.

10. Tester, J.W. and M. Modell, Thermodynamics and Its Applications. Prentice Hall International Series in the Physical and Chemical Engineering Sciences, ed. N.R. Amundson. 1997, Upper Saddle River, New Jersey: Prentice-Hall, Inc.

11. DeHoff, R.T., Thermodynamics in Materials Science. McGraw-Hill Series in Materials Science and Engineering, ed. B.J. Clark and J.M. Morriss. 1993, New York: McGraw-Hill, Inc.

12. Chakrabarti, C.G. and K. De, Boltzmann-Gibbs Entropy: Axiomatic Characterization and Application. International Journal of Mathematics and Mathematical Sciences, 2000. 23 (4): p. 243-251.

13. Shannon, C.E., A Mathematical Theory of Communication. Bell System Technical Journal, 1948. 27: p. 379-423, 623656.

14. Chakrabarti, C.G. and I. Chakrabarty, Shannon Entropy: Axiomatic Characterization and Application. International Journal of Mathematics and Mathematical Sciences, 2005. 17: p. 2847-2854.

15. Papoulis, A., Probability, Random Variables, and Stochastic Processes. 3rd ed. McGraw-Hill Series in Electrical Engineering, ed. S.W. Director. 1991, Singapore: McGraw-Hill, Inc.

16. Krykacz-Hausmann, B., Epistemic Sensitivity Analysis Based on the Concept of Entropy, in SAMO2001, P. Prado and R. Bolado, Editors. 2001, CIEMAT: Madrid. p. 31-35.

17. Jaynes, E.T., Information Theory and Statistical Mechanics. The Physical Review, 1957. 106(4): p. 620630.

18. Martyushev, L.M. and V.D. Seleznev, Maximum Entropy Production Principle in Physics, Chemistry and Biology. Physics Reports, 2006. 426: p. 1-45.

19. Turkay, S. and H. Akcay, A study of random vibration characteristics of the quarter-car model. Journal of Sound and Vibration, 2005. 282(1-2): p. 111-124.

20. Hac, A., Suspension Optimization of 2-dof Vehicle Model Using a Stochastic Optimal Control Technique'. Journal of Sound and Vibration, 1985. 100(3): p. 343-357.
21. Newland, D.E., An Introduction to Random Vibrations and Spectral Analysis. 4 ed. 1975, London and New York: Longman. 